\title{
Validação do questionário "Conocimiento del Paciente sobre sus Medicamentos” (CPM-ES-ES)
}

\author{
Validation of the "Conocimiento del Paciente sobre sus \\ Medicamentos" (CPM-ES-ES) questionnaire
}

Thiago Vinicius Nadaleto Didone (http://orcid.org/0000-0002-2397-5797) ${ }^{1}$

Pilar García-Delgado (https://orcid.org/0000-0003-1213-8165) ${ }^{2}$

Daniela Oliveira de Melo (https://orcid.org/0000-0001-8613-7953) ${ }^{3}$

Nicolina Silvana Romano-Lieber (http://orcid.org/0000-0002-8008-5547) ${ }^{4}$

Fernando Martínez Martínez (http://orcid.org/0000-0001-8247-1751) ${ }^{2}$

Eliane Ribeiro (https://orcid.org/0000-0003-0886-368X) ${ }^{1}$
${ }^{1}$ Faculdade de Ciências Farmacêuticas, Universidade de São Paulo (USP). Av. Prof. Lineu Prestes 580, Butantã. 05508-000 São Paulo SP Brasil. tdidone@gmail.com

${ }^{2}$ Facultad de Farmacia, Universidad de Granada.

Granada Espanha. ${ }^{3}$ Instituto de Ciências Ambientais, Químicas e Farmacêuticas, Universidade Federal de São Paulo. São Paulo SP Brasil

${ }^{4}$ Faculdade de Saúde Pública, USP. São Paulo SP Brasil.

\begin{abstract}
This work cross-culturally adapted the Spanish questionnaire 'Patients' knowledge about their medications ("Conocimiento del Paciente sobre sus Medicamentos" - CPM-ES-ES) for use in Brazil. It measures the level of medication knowledge by means of 11 questions. Eighty patients $\geq 80$ years were investigated and in 39 cases the caregivers were interviewed. The evaluation of conceptual and item equivalences considered the concept of knowledge and the questions that assess it as pertinent. Semantic equivalence was obtained by the correspondence in the denotative and connotative meaning of items. The study of measurement equivalence included factorial analysis and the calculation of validity and reliability estimates. As with the original questionnaire, principal component analysis identified 4 components, however, in 2 of them there were differences regarding included items. One question was removed from this analysis due to its sample inadequacy. Medication knowledge was correlated with medication regimen complexity $r=-.22$, $p=.046$. Medication knowledge of antihypertensives was correlated with their adherence $r=.70$, $p<.001$, and blood pressure control $r_{b}=.46, p$ $=.029$. The adapted version revealed functional equivalence, therefore it can be used in the Brazilian context.
\end{abstract}

Key words Cross-cultural comparison, Patient medication knowledge, Elderly, Questionnaires, Translations
Resumo Este trabalho adaptou transculturalmente o questionário espanhol "Conocimiento del Paciente sobre sus Medicamentos" (CPM-ES -ES) para uso no Brasil. Ele mede o grau de conhecimento sobre medicamentos por meio de 11 perguntas. Oitenta pacientes $\geq 80$ anos foram investigados e com 39 também foi entrevistado o cuidador. A avaliação das equivalências conceitual e de item considerou o conceito de conhecimento e as perguntas que o medem como pertinentes. A equivalência semântica foi obtida pela correspondência de significado denotativo e conotativo dos itens. O estudo da equivalência de mensuração incluiu análise fatorial e o cálculo de estimativas de validade e confiabilidade. Semelhante ao questionário original, a análise de componentes principais identificou 4 componentes, porém, em 2 deles houve diferenças nos itens incluídos. Uma pergunta foi removida desta análise devido à sua inadequação amostral. O conhecimento sobre medicamentos esteve correlacionado à complexidade da prescrição $r=-0,22, p=0,046$. O conhecimento sobre anti-hipertensivos esteve correlacionado à sua adesão $r=0,70, p<0,001$, e ao controle da pressão arterial $r_{b}=0,46, p=0,029$. A versão adaptada apresentou equivalência funcional de modo que pode ser usada no contexto brasileiro.

Palavras-chave Comparação transcultural, Conhecimento do paciente sobre a medicação, Idoso, Questionários, Traduções 


\section{Introdução}

O uso racional de medicamentos compreende a prescrição e a dispensação adequadas, a disponibilidade oportuna e a preços acessíveis, e o consumo nas doses, intervalos e períodos indicados de medicamentos eficazes, seguros e de qualida$\mathrm{de}^{1}$. Não obstante, a efetividade do medicamento depende ultimamente de que seu usuário siga as instruções recebidas. Nesse contexto, pacientes precisam receber informações para garantir conhecimentos e habilidades necessários a utilizar medicamentos apropriadamente ${ }^{2}$. A promoção do uso racional de medicamentos envolve a orientação do usuário quanto à indicação terapêutica e posológica, aos cuidados gerais de administração, às interações medicamentosas, e aos riscos e ao gerenciamento de reações adversas, sobredose e descontinuação do medicamento ${ }^{3}$.

Estudos de base populacional evidenciaram a alta medicalização de idosos e reafirmaram a importância da avaliação dos medicamentos por eles utilizados com o objetivo de promover seu uso racional ${ }^{4,5}$. Nos municípios de São Paulo e Campinas, SP, as frequências de idosos que usavam no mínimo um medicamento foram, respectivamente, $89,6 \%{ }^{4}$ e $80,4 \%{ }^{5}$; em relação aos idosos que usavam no mínimo cinco medicamentos, as frequências foram, respectivamente, $36,0 \%{ }^{4}$ e $24,8 \%{ }^{5}$.Além disso, pacientes idosos costumam apresentar um conhecimento sobre medicamentos insuficiente ${ }^{6-8}$ e menor em relação a pacientes mais joven $s^{8,9}$. Quando insuficiente, $o$ conhecimento do paciente sobre o medicamento (CPM) pode resultar na não adesão farmacoterapêutica ${ }^{6,10}$ e na piora clínica ${ }^{9}$.

Com exceção dos trabalhos cujo objetivo principal é medi-lo,o CPMé raramente conceituado, definido ou categorizado. Para Silva et al. ${ }^{11}$, ele foi explicado por sete perguntas que investigaram informações sobre o nome, dose, frequência de administração, indicação, duração do tratamento, efeitos adversos e precauções. As respostas foram categorizadas entre corretas ou incorretas. Embora não houvessem mencionado o texto de algumas perguntas e a maneira de pontuá-las, o método utilizado pelos autores foi reproduzido com ou sem modificações em outros trabalhos ${ }^{12-15}$. Alguns autores ampliarameste conceito adicionando questões sobre a forma de administração, interações e procedimento a ser tomado no caso de esquecimento dedoses, e, ain$\mathrm{da}$, uma outra categoria de resposta: não sabe ${ }^{12}$. Apesar da grande contribuição desses trabalhos para o estudo do CPM, nenhum deles realizou testes de confiabilidade ou validade nos questionários usados.Desse modo, o Brasil ainda carece de um questionário com estes tipos de avaliações para medir o CPM.

Delgado et al..$^{16}$ definiu CPM como:

o conjunto de informações adquiridas pelo paciente sobre seu medicamento necessário para o seu uso correto, que inclui o objetivo terapêutico (indicação e efetividade), o processo de uso (dose, frequência, duração do tratamento, e forma de administração), a segurança (precauções, reações adversas, contraindicações e interações) e a conservação.

Baseado nessa definição, foi criado o questionário "Conocimiento del Paciente sobre sus Medicamentos" (CPM-ES-ES), um instrumento espanhol válido para medir o conhecimento que alguém possua sobre os medicamentos que utiliza. Ele possui 11 perguntas divididas em quatro componentes: processo de uso, objetivo terapêutico, segurança e conservação. Cada resposta é pontuada e o conhecimento é calculado pela média ponderada dos escores ${ }^{16,17}$.

Embora este questionário tenha sido adaptado ao português europeu ${ }^{18}$, julgou-se necessária sua adaptação também ao português brasileiro, justificado pelas diferenças culturais entre Portugal e Brasil. Desse modo, foi realizada a adaptação transcultural do questionário CPM-ES-ES em pacientes idosos ambulatoriais com $\geq 80$ anos, ou seus cuidadores, a fim de se propor uma versão em língua portuguesa, para uso no Brasil, que pudesse mensurar o CPM.

\section{Métodos}

\section{Etapas}

A adaptação transcultural (ATC) foi realizada para o questionário CPM-ES-ES e avaliou as equivalências conceitual, de item, semântica, operacional, de mensuração, e funcional ${ }^{19}$.

\section{Equivalência conceitual e de item}

A equivalência conceitual foi baseada na capacidade dos domínios e dos seus pesos, contemplados no instrumento original, em representar de maneira adequada o conceito de CPM no contexto brasileiro. A equivalência de itens baseou-se na apreciação da pertinência das perguntas (itens)em mensurar na cultura brasileira os domínios descritos originalmente ${ }^{19}$. Ambas foram avaliadas simultaneamente por dois farmacêuticos com experiência em cuidados farmacêuticos por meio de discussões. Para tanto, fo- 


\section{População e local de estudo}

Foram entrevistados idosos, ou seus cuidadores, atendidos no Ambulatório de Fragilidade do Hospital Universitário da Universidade de São Paulo (AF/HU/USP) de março/2013 a fevereiro/2014, de modo consecutivo. O referido ambulatório conta com geriatras que prestam atendimento a idosos com $\geq 80$ anos e, no mínimo, uma das seguintes condições: uso de dispositivo auxiliar de marcha, uso de cinco medicamentos ou mais, demência, doença de Parkinson, duas ou mais quedas no último ano, ou funcionalidade reduzida. As entrevistas foram realizadas imediatamente após a consulta com o geriatra. Caso o paciente apresentasse diagnóstico de demência reportado em prontuário ou necessitasse de ajuda para usar seus medicamentos, optou-se por entrevistar o cuidador responsável por administrar seus medicamentos. No AF/HU/USP, os critérios diagnósticos de demência são os descritos no Manual Diagnóstico e Estatístico de Doenças Mentais $^{20}$.

Os critérios de inclusão foram:

Comparecimento à consulta médica;

Prescrição de um ou mais medicamentos;

Presença do cuidador responsável por administrar os medicamentos.

Foram excluídos pacientes que receberam atendimento de urgência ou que se recusassem a participar do estudo.

\section{Coleta de dados}

Coletaram-se as seguintes informações dos respondentes: idade, sexo, nível de instrução, e rendimento mensal. Dos pacientes coletaram-se dados do controle da pressão arterial, da adesão à farmacoterapia anti-hipertensiva, e das doenças descritas em prontuário. Dos medicamentos prescritos foram registrados: nome, número, tempo de uso, complexidade da prescrição,e CPM. Todos os dados referem-se à prescrição fornecida na consulta precedente à entrevista, a qual podia incluir desde medicamentos prescri-

tos por muitos anos até aqueles prescritos pela primeira vez.

Pacientes hipertensos tiveram sua pressão arterial (PA) medida em pré-consulta de enfermagem utilizando um aparelho semiautomático digital de braço validado e calibrado. Tanto a mensuração da PA como o diagnóstico de hipertensão no $\mathrm{AF} / \mathrm{HU} / \mathrm{USP}$ foram realizados de acordo com as recomendações das VI Diretrizes Brasileiras de Hipertensão ${ }^{21}$. Aqueles com PA sistólica $\leq 145 \mathrm{mmHg}^{22}$ foram considerados controlados, enquanto os demais foram considerados não controlados. A adesão aos anti-hipertensivos foi representada pela porcentagem da dose diária utilizada em relação à dose diária prescrita para cada medicamento; aqueles com porcentagens entre $80 \%$ e $120 \%$ são pontuados com 1; os demais recebem 0 . A soma dos pontos dividida pelo número total de medicamentos prescritos representa a adesão, que pode variar de $0 \mathrm{a} 1^{6}$. A adesão não foi avaliada para medicamentos cujo nome o entrevistado desconhecia nem para aqueles prescritos pela primeira vez.

A complexidade da prescrição foi avaliada por meio de instrumento adaptado ao português brasileiro que mede o Índice de Complexidade da Farmacoterapia (ICFT) para um paciente individual. Ele é calculado pela soma dos pontos obtidos em três características relacionadas aos medicamentos: suas formas farmacêuticas, as frequências de dose prescritas e as informações de uso presentes na receita médica. Quanto maior a capacidade da característica em aumentar a complexidade da administração dos medicamentos, maior a sua pontuação. Assim, tanto maior será a complexidade da prescrição quanto maior for o valor do ICFT ${ }^{23}$.

No presente estudo, obteve-se um valor de CPM para cada medicamento prescrito por meio da aplicação do questionário aqui adaptado. Permitiu-se ao entrevistado ler a prescrição, caso desejasse, para responder às questões. Medicamentos cujo nome o entrevistado desconhecia foram classificados como ausência de conhecimento $(\mathrm{CPM}=0)$.

O CPM-ES-ES possui 11 perguntas abertas divididas em quatro componentes (Quadro 1). O primeiro, denominado processo de uso, é representado pelas perguntas $2,3,4$ e 5 , as quais investigam respectivamente o conhecimento sobre dose, frequência, duração do tratamento e forma de administração. O segundo, objetivo terapêutico, abrange as perguntas 1 e 9 , as quais investigam respectivamente o conhecimento acerca da indicação e da efetividade. O terceiro, 
Quadro 1. Versão original do questionário CPM-ES-ES e sua versão adaptada ao português brasileiro com sugestões de melhoria.

\begin{tabular}{|c|c|c|c|}
\hline Pergunta & $\begin{array}{l}\text { Informações } \\
\text { investigadas }\end{array}$ & $\begin{array}{c}\text { Versão original em espanhol } \\
\text { (CPM-ES-ES) }\end{array}$ & $\begin{array}{c}\text { Versão adaptada ao português } \\
\text { brasileiro }^{\text {a }}\end{array}$ \\
\hline & & $\begin{array}{c}\text { Conocimiento del Paciente sobre sus } \\
\text { Medicamentos }\end{array}$ & $\begin{array}{c}\text { Conhecimento do Paciente sobre } \\
\text { seus Medicamentos }\end{array}$ \\
\hline 1 & Indicação & $\begin{array}{l}\text { ¿Para qué tiene que tomar este } \\
\text { medicamento? }\end{array}$ & $\begin{array}{l}\text { Para que tem/terá que tomar/utilizar } \\
\text { este medicamento? }\end{array}$ \\
\hline 2 & Dose & $\begin{array}{l}\text { ¿Qué cantidad debe tomar/utilizar de } \\
\text { este medicamento? }\end{array}$ & $\begin{array}{l}\text { Qual a quantidade que deve(rá) } \\
\text { tomar/utilizar deste medicamento? }\end{array}$ \\
\hline 3 & Frequência & $\begin{array}{l}\text { ¿Cada cuánto tiene que tomar/utilizar } \\
\text { este medicamento? }\end{array}$ & $\begin{array}{l}\text { Quantas vezes ao dia tem/terá que } \\
\text { tomar/utilizar este medicamento? }\end{array}$ \\
\hline 4 & $\begin{array}{l}\text { Duração do } \\
\text { tratamento }\end{array}$ & $\begin{array}{l}\text { ¿Hasta cuándo tiene que tomar/utilizar } \\
\text { este medicamento? }\end{array}$ & $\begin{array}{l}\text { Até quando tem/terá que tomar/ } \\
\text { utilizar este medicamento? }\end{array}$ \\
\hline 5 & $\begin{array}{l}\text { Forma de } \\
\text { administração }\end{array}$ & $\begin{array}{l}\text { ¿Cómo debe tomar/utilizar este } \\
\text { medicamento? }\end{array}$ & $\begin{array}{l}\text { Como deve(rá) tomar/utilizar este } \\
\text { medicamento? }\end{array}$ \\
\hline 6 & Precauções & $\begin{array}{l}\text { ¿Ha de tener alguna precaución cuándo } \\
\text { toma/utiliza este medicamento? }\end{array}$ & $\begin{array}{l}\text { Deve(rá) ter algum cuidado quando } \\
\text { toma(r)/utiliza(r) este medicamento? }\end{array}$ \\
\hline 7 & Reações adversas & $\begin{array}{l}\text { ¿Qué efectos adversos conoce usted de } \\
\text { este medicamento? }\end{array}$ & $\begin{array}{l}\text { Quais reações adversas você conhece } \\
\text { deste medicamento? }\end{array}$ \\
\hline 8 & Contraindicações & $\begin{array}{l}\text { ¿Ante qué problema de salud o situación } \\
\text { especial no debe tomar/utilizar este } \\
\text { medicamento? }\end{array}$ & $\begin{array}{l}\text { Diante de qual problema de saúde ou } \\
\text { situação especial não deve(rá) tomar/ } \\
\text { utilizar este medicamento? }\end{array}$ \\
\hline 9 & Efetividade & $\begin{array}{l}\text { ¿Cómo sabe si el medicamento le hace } \\
\text { efecto? }\end{array}$ & $\begin{array}{l}\text { Como sabe se o medicamento fazl } \\
\text { fará efeito? }\end{array}$ \\
\hline 10 & Interações & $\begin{array}{l}\text { ¿Qué medicamentos o alimentos } \\
\text { debe evitar tomar mientras use este } \\
\text { medicamento? }\end{array}$ & $\begin{array}{l}\text { Quais medicamentos ou alimentos } \\
\text { devem/deverão ser evitados durante } \\
\text { o uso deste medicamento? }\end{array}$ \\
\hline 11 & Conservação & ¿Cómo debe conservar su medicamento? & $\begin{array}{l}\text { Como deve(rá) conservar este } \\
\text { medicamento? }\end{array}$ \\
\hline
\end{tabular}

${ }^{a}$ As sugestões de melhoria estão grafadas em negrito e tem a intenção de facilitar a investigação do conhecimento de medicamentos prescritos pela primeira vez e não administrados por via oral.

segurança, é composto pelas perguntas $6,7,8 \mathrm{e}$ 10 , as quais avaliam o conhecimento sobre precauções, reações adversas, contraindicações e interações respectivamente. O quarto, conservação, é representado pela pergunta 11 , que investiga o conhecimento da conservação. Cada resposta é pontuada segundo sua concordância com a informação de referência. Respostas incorretas recebem -1; entrevistados que declaram verbal ou não verbalmente não saber a resposta recebem 0; respostas incompletas, isto é, aquelas que não permitem assegurar o uso correto e seguro do medicamento, recebem 1; respostas corretas, ou seja, aquelas que correspondem com exatidão às informações de referência, recebem 2. O CPM é a média ponderada dos escores obtidos nas 11 questões, segundo os pesos a seguir; perguntas do domínio processo de uso: peso 1,2; perguntas do objetivo terapêutico: peso 1,1; perguntas do segurança: peso 0,85; pergunta do conservação: peso 0,6. Recomenda-se classificar o CPM nas categorias: ausência de conhecimento (0), conhecimento insuficiente $(0,60$ a 1,26$)$, conhecimento suficiente $(1,27$ a 1,60$)$, e conhecimento ótimo $(1,61 \text { a } 2,00)^{16,17}$.

Foram consideradas de referência as informações da prescrição para as perguntas do domínio processo de uso, e dos bancos de dados Micromedex $^{24}$ e UpToDate ${ }^{25}$ para as perguntas dos outros domínios. As informações investigadas pela pergunta 4 não estavam presentes em muitas prescrições. Assim, nos casos em que essa informação estava presente, a prescrição foi considerada a informação de referência; quando ausente, considerou-se como referência os bancos de dados supracitados. 


\section{Equivalência semântica e operacional}

A obtenção da equivalência semântica seguiu as etapas descritas a seguir. Duas traduções para o português foram realizadas independentemente por dois brasileiros nativos, fluentes no idioma espanhol, um pertencente e outro leigo à área da saúde, os quais construíram T-1 e T-2 respectivamente. Tais versões deram origem a uma versão consensual de tradução, T-12, construída pelos farmacêuticos. Esta foi retraduzida ao espanhol de modo independente e cego em relação aos indivíduos que atuaram na etapa inicial, por dois tradutores que possuem o espanhol como idioma nativo, leigos à área da saúde, resultando em duas versões de retradução, R-1 e R-2. Em uma etapa seguinte, os farmacêuticos compararam as versões de retradução com o questionário original a fim de avaliar a semelhança denotativa e conotativa entre as palavras, assim como as reações que as mesmas poderiam desencadear quando utilizadas na cultura brasileira ${ }^{26}$. Eventuais divergências de significado foram discutidas e uma versão pré-final do questionário foi concebida de modo que pudesse ser compreendida por qualquer pessoa fluente em português do Brasil, com nível de instrução correspondente ao sexto ano primário $^{27}$. Sua compreensão foi avaliada por um pré-teste em que 30 indivíduos (18 pacientes e 12 cuidadores) foram indagados sobre o entendimento das questões. As entrevistas do pré-teste permitiram aos farmacêuticos avaliar a equivalência operacional. Para tanto, verificaram a adequação do veículo, formato, modo e ambiente de aplicação das perguntas, e da técnica de categorização das respostas com vistas a mensurar o $\mathrm{CPM}^{19}$.

\section{Equivalência de mensuração e funcional}

Após o pré-teste, outros 50 indivíduos foram entrevistados. Assim, a avaliação da equivalência de mensuração valeu-se dos dados coletados de 80 indivíduos entrevistados (41 pacientes e 39 cuidadores).Foram utilizados três enfoques psicométricos: avaliação exploratória da estrutura interna,da confiabilidade dos dados obtidos, e da validade do instrumento.

A estrutura interna foi obtida por análise de componentes principais (ACP) considerando um medicamento prescrito por paciente, cuja escolha ocorreu de forma aleatória $(\mathrm{N}=80)$.A lista completa dos medicamentos e suas frequências estão na Tabela 1. Para verificar a adequação do tamanho amostral para a ACP foi utilizado o
Tabela 1. Frequência dos medicamentos utilizados na análise de componentes principais e no cálculo do alfa de Cronbach, N=80, São Paulo-SP, 2013-2014.

\begin{tabular}{|c|c|}
\hline Medicamento & $\mathbf{n}$ \\
\hline Ácido acetilsalicílico & 7 \\
\hline Enalapril & 7 \\
\hline Vitamina D & 5 \\
\hline Atenolol & 4 \\
\hline Omeprazol & 4 \\
\hline
\end{tabular}

Anlodipino

Captopril

Donepezila

Losartana

Hidroclorotiazida

Levotiroxina

Metformina

Oxibutinina

Vitaminas A e D

Sinvastatina

Alendronato

Alopurinol

Cálcio

Cálcio e vitamina D

Clopidogrel

Digoxina

Diltiazem

Domperidona

Espironolactona

Fluconazol

Formoterol

Furosemida

Galantamina

Glibenclamida

Gliclazida

Hidroclorotiazida e amilorida

Hidroxicloroquina

Insulina isófona $(\mathrm{NPH})$

Memantina

Ondansetrona

Paracetamol

Sertralina

Sorbitol e lauril sulfato de sódio

Tansulosina

Vitaminas B1, B6 e B12

Tiocolchicosídeo 1

Trazodona 1

Varfarina 1

Viminol

1

índice de Kaiser-Meyer-Olkin (KMO), onde valores abaixo de 0,5 indicam má adequação ${ }^{28}$. Posteriormente, valores altos (se $>0,8)$ e baixos (se $<$ 
0,3) na matriz de correlação entre itens e a determinante da matriz (se $<0,00001$ ) foram avaliados para identificar problemas na separação dos componentes $^{29}$. Para identificar a significância da média das correlações (se igual ou diferente de 0) foi aplicado o teste de esfericidade de Bartlett ${ }^{29}$. Itens com KMO inadequado e com problemas na separação dos componentes segundo a avaliação das correlações foram considerados inadequados para a ACP, excluídos e realizada nova ACP exploratória. As comunalidades, que representam a proporção de variância comum para cada item, podem indicar a adequação do método usado para escolher o número de componentes extraídos na $\mathrm{ACP}^{29}$. A extração dos componentes foi pré-determinada em 4 componentes, como na construção original da escala ${ }^{16}$, com rotação $v a$ rimax na avaliação dos pesos fatoriais. Valores $<0,40$ nos pesos fatoriais foram considerados como itens não significativos ${ }^{30}$. Os componentes da ACP final foram determinados pelos itens com maior carga fatorial absoluta ${ }^{29,31}$. A variância explicada foi obtida da proporção da variância comum entre os componentes e os itens.

O coeficiente alfa de Cronbach $(\alpha)$ foi utilizado para quantificar a consistência interna dos itens $^{31}$, uma estimativa da confiabilidade. Valores iguais ou acima de 0,70 foram considerados adequados $^{32}$.

A validade discriminante foi estimada pelo coeficiente de correlação de Pearson (r) entre a mediana dos valores de CPM e a complexidade da prescrição $(\mathrm{N}=80)$.

A validade convergente foi estimada pelos coeficientes de correlação de Pearson (r) e bisserial $\left(\mathrm{r}_{\mathrm{b}}\right)$ dos valores medianos de CPM dos anti-hipertensivos com a adesão e o controle de pressão arterial, respectivamente. Adicionalmente, foram realizados gráficos de dispersão. Foram considerados somente os pacientes hipertensos $(n=61)$, visto que foi a doença mais prevalente na amostra e todos os pacientes possuíam a prescrição de no mínimo um anti-hipertensivo.

A equivalência funcional foi obtida pelos farmacêuticos por meio de discussões, a fim de avaliar a extensão pela qual o questionário mede o CPM, considerando a adequação dos diferentes tipos de equivalência, consistência interna e validade ${ }^{19}$.

A análise estatística foi realizada no software IBM Statistical Package for Social Sciences versão 20(IBM Corporation, Armonk, NY, USA). P-valores foram considerados significativos quando $<0,050$. Intervalos de confiança percentílico bootstrap corrigido do viés e acelerado de 95\%
(IC95\% BCa) foram calculados para os coeficientes de correlação.

\section{Ética}

O projeto foi aprovado pelos Comitês de Ética em Pesquisa da Faculdade de Ciências Farmacêuticas e do HU da USP, e todos os entrevistados assinaram um Termo de Consentimento Livre e Esclarecido.

\section{Resultados}

\section{Equivalência conceitual e de item}

A BIREME define o CPM como o conhecimento de saúde do paciente relacionado a medicamentos incluindo o que está sendo usado e o porquê, bem como instruções e precauções ${ }^{33}$. Segundo estudos brasileiros, quando o CPM é suficiente, representa o conhecimento necessário para que o paciente tenha condições de utilizar o medicamento de forma segura ${ }^{11-15}$. Os estudos brasileiros utilizaram sete ${ }^{11,14,15}$, oito ${ }^{13}$ ou nove $e^{12}$ perguntas para medir o conhecimento, e investigaram informações sobre o processo de uso, segurança e indicação. Ainda, perguntas sobre o processo de uso do medicamento tinham geralmente um peso maior no cálculo do escore de conhecimento, uma vez que foram consideradas as mais importantes para seu uso seguro ${ }^{11-15}$.

Assim, os farmacêuticos consideraram que o conceito de $\mathrm{CPM}^{16}$, os quatro domínios que o compõem, e seus pesos, são semelhantes ou mais abrangentes que aqueles descritos por estudo nacionais e, portanto, permitem representar e mensurar adequadamente o CPM no contexto cultural brasileiro. Por exemplo, o questionário original investiga informações sobre conservação e efetividade do tratamento, ausentes nas publicações nacionais. Além disso, as perguntas foram consideradas fundamentais e suficientes para a avaliação dos domínios, podendo ser usadas sem outra modificação que a tradução. Assim, concluiu-se haver plena equivalência conceitual e de item entre o questionário original e o adaptado, os quais são apresentados no Quadro 1. A versão adaptada incluiu sugestões de melhoria.

\section{Equivalência semântica e operacional}

As versões de tradução, retradução, e a versão consensual de tradução encontram-se no quadro 2. Durante a elaboração de T-12, os farmacêuticos 
Quadro 2. Versões de tradução, retradução, e versão consensual de tradução que compuseram as etapas para a obtenção da equivalência semântica.

\begin{tabular}{|c|c|c|c|c|c|}
\hline Pergunta & T-1 & T-2 & T-12 & R-1 & R-2 \\
\hline 1 & $\begin{array}{l}\text { Para que tem } \\
\text { que tomar este } \\
\text { medicamento? }\end{array}$ & $\begin{array}{l}\text { Para que toma esse } \\
\text { medicamento? }\end{array}$ & $\begin{array}{l}\text { Para que tem } \\
\text { que tomar este } \\
\text { medicamento? }\end{array}$ & $\begin{array}{l}\text { ¿Para qué tiene } \\
\text { que tomar este } \\
\text { medicamento? }\end{array}$ & $\begin{array}{l}\text { ¿Para qué tienes } \\
\text { que tomar este } \\
\text { medicamento? }\end{array}$ \\
\hline 2 & $\begin{array}{l}\text { Que quantidade } \\
\text { deve tomar/ } \\
\text { utilizar deste } \\
\text { medicamento? }\end{array}$ & $\begin{array}{l}\text { Qual a quantidade } \\
\text { que toma/ } \\
\text { utiliza desse } \\
\text { medicamento? }\end{array}$ & $\begin{array}{l}\text { Qual a quantidade } \\
\text { que deve tomar/ } \\
\text { utilizar deste } \\
\text { medicamento? }\end{array}$ & $\begin{array}{l}\text { ¿Qué cantidad de } \\
\text { este medicamento } \\
\text { debe tomar/ } \\
\text { utilizar? }\end{array}$ & $\begin{array}{l}\text { ¿Qué cantidad de } \\
\text { este medicamento } \\
\text { debes tomar/ } \\
\text { utilizar? }\end{array}$ \\
\hline 3 & $\begin{array}{l}\text { De quanto em } \\
\text { quanto tempo } \\
\text { tem que tomar/ } \\
\text { utilizar este } \\
\text { medicamento? }\end{array}$ & $\begin{array}{l}\text { Quantas vezes } \\
\text { ao dia tem que } \\
\text { tomar/utilizar esse } \\
\text { medicamento? }\end{array}$ & $\begin{array}{l}\text { Quantas vezes } \\
\text { ao dia tem que } \\
\text { tomar/utilizar este } \\
\text { medicamento? }\end{array}$ & $\begin{array}{l}\text { ¿Cuántas veces } \\
\text { al día tiene } \\
\text { que tomar/ } \\
\text { utilizar este } \\
\text { medicamento? }\end{array}$ & $\begin{array}{l}\text { ¿Cuántas veces al } \\
\text { día debes tomar/ } \\
\text { utilizar este } \\
\text { medicamento? }\end{array}$ \\
\hline 4 & $\begin{array}{l}\text { Até quando } \\
\text { tem que tomar/ } \\
\text { utilizar este } \\
\text { medicamento? }\end{array}$ & $\begin{array}{l}\text { Até quando tem } \\
\text { que tomar/utilizar } \\
\text { esse medicamento? }\end{array}$ & $\begin{array}{l}\text { Até quando } \\
\text { tem que tomar/ } \\
\text { utilizar este } \\
\text { medicamento? }\end{array}$ & \begin{tabular}{|l} 
¿Hasta cuándo \\
tiene que tomar/ \\
utilizar este \\
medicamento?
\end{tabular} & $\begin{array}{l}\text { ¿Hasta cuándo } \\
\text { debes tomar/ } \\
\text { utilizar este } \\
\text { medicamento? }\end{array}$ \\
\hline 5 & $\begin{array}{l}\text { Como deve } \\
\text { tomar/utilizar este } \\
\text { medicamento? }\end{array}$ & $\begin{array}{l}\text { Como deve tomar/ } \\
\text { utilizar esse } \\
\text { medicamento? }\end{array}$ & $\begin{array}{l}\text { Como deve } \\
\text { tomar/utilizar este } \\
\text { medicamento? }\end{array}$ & $\begin{array}{l}\text { ¿Cómo } \\
\text { debe tomar/ } \\
\text { utilizar este } \\
\text { medicamento? }\end{array}$ & $\begin{array}{l}\text { ¿Cómo } \\
\text { debes tomar/ } \\
\text { utilizar este } \\
\text { medicamento? }\end{array}$ \\
\hline 6 & $\begin{array}{l}\text { Tem que tomar } \\
\text { alguma precaução } \\
\text { quando tomar/ } \\
\text { utiliza este } \\
\text { medicamento? }\end{array}$ & $\begin{array}{l}\text { É preciso ter } \\
\text { algum cuidado } \\
\text { quando toma/ } \\
\text { utiliza esse } \\
\text { medicamento? }\end{array}$ & $\begin{array}{l}\text { Deve ter algum } \\
\text { cuidado quando } \\
\text { toma/utiliza este } \\
\text { medicamento? }\end{array}$ & $\begin{array}{l}\text { ¿Debe tomar } \\
\text { algún cuidado } \\
\text { al tomar/ } \\
\text { utilizar este } \\
\text { medicamento? }\end{array}$ & $\begin{array}{l}\text { ¿Debes tomar } \\
\text { algún cuidado } \\
\text { al tomar/ } \\
\text { utilizar este } \\
\text { medicamento? }\end{array}$ \\
\hline 7 & $\begin{array}{l}\text { Que efeitos } \\
\text { colaterais você } \\
\text { conhece deste } \\
\text { medicamento? }\end{array}$ & $\begin{array}{l}\text { Quais reações } \\
\text { adversas você } \\
\text { conhece desse } \\
\text { medicamento? }\end{array}$ & $\begin{array}{l}\text { Quais reações } \\
\text { adversas você } \\
\text { conhece deste } \\
\text { medicamento? }\end{array}$ & $\begin{array}{l}\text { ¿Cuáles } \\
\text { reacciones } \\
\text { adversas a este } \\
\text { medicamento } \\
\text { usted conoce? }\end{array}$ & $\begin{array}{l}\text { ¿Cuáles } \\
\text { reacciones } \\
\text { adversas a este } \\
\text { medicamento } \\
\text { conoces? }\end{array}$ \\
\hline 8 & $\begin{array}{l}\text { Diante de qual } \\
\text { problema ou } \\
\text { situação especial } \\
\text { não deve tomar/ } \\
\text { utilizar este } \\
\text { medicamento? }\end{array}$ & $\begin{array}{l}\text { Esse medicamento } \\
\text { não deve ser } \\
\text { tomado/utilizado } \\
\text { diante de qual } \\
\text { problema de } \\
\text { saúde ou situação } \\
\text { especial? }\end{array}$ & $\begin{array}{l}\text { Diante de qual } \\
\text { problema de } \\
\text { saúde ou situação } \\
\text { especial não deve } \\
\text { tomar/utilizar este } \\
\text { medicamento? }\end{array}$ & $\begin{array}{l}\text { ¿En cuál } \\
\text { problema de } \\
\text { salud o situación } \\
\text { especial no } \\
\text { debe tomar/ } \\
\text { utilizar este } \\
\text { medicamento? }\end{array}$ & $\begin{array}{l}\text { ¿En cuál } \\
\text { problema de } \\
\text { salud o situación } \\
\text { especial no } \\
\text { debes tomar/ } \\
\text { utilizar este } \\
\text { medicamento? }\end{array}$ \\
\hline 9 & $\begin{array}{l}\text { Como sabe se o } \\
\text { medicamento fez } \\
\text { efeito? }\end{array}$ & $\begin{array}{l}\text { Como sabe se o } \\
\text { medicamento tem } \\
\text { efeito? }\end{array}$ & $\begin{array}{l}\text { Como sabe se o } \\
\text { medicamento faz } \\
\text { efeito? }\end{array}$ & $\begin{array}{l}\text { ¿Cómo sabe si } \\
\text { el medicamento } \\
\text { produce efecto? }\end{array}$ & $\begin{array}{l}\text { ¿Cómo sabes si } \\
\text { el medicamento } \\
\text { produce efecto? }\end{array}$ \\
\hline 10 & $\begin{array}{l}\text { Que } \\
\text { medicamentos } \\
\text { ou alimentos } \\
\text { devem evitar } \\
\text { enquanto usa este } \\
\text { medicamento? }\end{array}$ & $\begin{array}{l}\text { Quais } \\
\text { medicamentos } \\
\text { ou alimentos } \\
\text { devem ser } \\
\text { evitados durante } \\
\text { o uso desse } \\
\text { medicamento? }\end{array}$ & $\begin{array}{l}\text { Quais } \\
\text { medicamentos } \\
\text { ou alimentos } \\
\text { devem ser } \\
\text { evitados durante } \\
\text { o uso deste } \\
\text { medicamento? }\end{array}$ & $\begin{array}{l}\text { ¿Cuáles } \\
\text { medicamentos } \\
\text { o alimentos } \\
\text { no deben ser } \\
\text { consumidos } \\
\text { mientras use este } \\
\text { medicamento? }\end{array}$ & $\begin{array}{l}\text { ¿Cuáles } \\
\text { medicamentos } \\
\text { o alimentos } \\
\text { no deben ser } \\
\text { consumidos } \\
\text { mientras uses este } \\
\text { medicamento? }\end{array}$ \\
\hline 11 & $\begin{array}{l}\text { Como deve ser a } \\
\text { conservação do } \\
\text { seu medicamento? }\end{array}$ & $\begin{array}{l}\text { Como esse } \\
\text { medicamento deve } \\
\text { ser conservado? }\end{array}$ & $\begin{array}{l}\text { Como deve } \\
\text { conservar este } \\
\text { medicamento? }\end{array}$ & $\begin{array}{l}\text { ¿Cómo debe } \\
\text { conservar este } \\
\text { medicamento? }\end{array}$ & $\begin{array}{l}\text { ¿Cómo debes } \\
\text { conservar este } \\
\text { medicamento? }\end{array}$ \\
\hline
\end{tabular}

T-1 = versão de tradução 1; T-2 = versão de tradução 2; T-12 = versão consensual de tradução; R-1 = versão de retradução 1 ; R-2 = versão de retradução 2 . 
optaram pelos itens das versões de tradução que mais se aproximavam da tradução literal. Os itens 1,4 e 5 foram incorporados integralmente de $\mathrm{T}-1$. Os itens 8 e 9, de T-1, e os itens 2, 3, 6, 7 e 10, de $\mathrm{T}-2$, foram incorporados com pequenas modificações.Já o item 11 foi completamente reescrito.

Quando comparadas à versão original, as questões 1, 2, 4, 5, 8, 9 e 11 de R-1 e R-2 possuíam o mesmo sentido denotativo e conotativo devido, sobretudo, à semelhança literal entre elas. Na questão 10, embora R-1 e R-2 não tivessem o mesmo sentido denotativo, julgou-se possuírem o mesmo sentido conotativo, e preferiu-se não alterar T-12. Nas questões 3, 6 e 7, embora tenha sido necessário substituir expressões literais por termos mais adequados, os farmacêuticos julgaram que o significado conotativo foi conservado.

Durante o pré-teste nenhum entrevistado se recusou ou deixou de responder a alguma pergunta, ou relatou dificuldades na sua compreensão.Assim, T-12 foi submetida, sem alterações, à avaliação psicométrica, e corresponde à versão adaptada ao português brasileiro do questionário CPM-ES-ES. Ainda, os farmacêuticos concluíram que o método de aplicação do questionário e de avaliação das respostas foram adequados para mensurar o CPM, podendo ser empregados sem alterações. Por tudo isso, concluiu-se haver equivalência semântica e operacional.

\section{Equivalência de mensuração e funcional}

As características dos entrevistados estão na Tabela 2. Hipertensão (76,3\%), hiperlipidemias $(32,5 \%)$, insuficiência cardíaca $(32,5 \%)$ e demências $(31,3 \%)$ foram as doenças mais frequentes. Ao todo, foram prescritos 496 medicamentos. Ácido acetilsalicílico (42,5\%), omeprazol $(36,3 \%)$, enalapril $(30,0 \%)$ e sinvastatina $(27,5 \%)$ configuraram como os mais prescritos. No que diz respeito ao tempo de uso, $76,2 \%$ dos medicamentos estavam sendo usados há mais de 6 meses, 9,5\% há menos de 6 meses, e 14,3\% nunca haviam sido usados (prescritos pela primeira vez).

A Tabela 3 apresenta o resultado de duas ACP (sem e com exclusão do item 8) e inclui os pesos fatoriais de cada pergunta, os autovalores e a variância explicada de cada componente. Em ambas análises, os itens agrupados no mesmo componente sugerem que o primeiro componente (perguntas 2, 3, 4 e 5) represente o conhecimento sobre as informações do processo de uso do medicamento e o segundo (perguntas 9 e 1) as informações acerca do objetivo terapêutico, ocu- pando a mesma localização na versão original. A diferença entre as ACP está na localização do item 7, presente no terceiro componente, na ACP com todos os itens, e no quarto, na ACP com exclusão do item 8 .

$\mathrm{Na}$ primeira ACP, a pergunta 8 apresentou correlações com outras perguntas variando de 0 a 0,3 e KMO individual de 0,439 , o que indicou sua exclusão e uma nova ACP exploratória com 10 perguntas. Esta ACP apresentou valores de $\mathrm{KMO}$ individuais $>0,529$ e KMO global de 0,778 . As correlações não apresentaram valores altos ou baixos, a determinante da matriz foi de 0,054 e o teste de Bartlett foi significativo ( $\mathrm{p}<$ $0,001)$. As comunalidades após a extração variaram de 0,570 a 0,792. Os quatro componentes extraídos apresentaram variância explicada total de $70,35 \%$.

Com relação à dispersão dos escores do item 8, 96,3\% (77/80) das respostas pontuaram zero. $\mathrm{O} \alpha$ foi 0,741 para todas as perguntas e 0,748 após a exclusão da pergunta 8 .

O CPM correlacionou-se negativamente ao ICFT ( $\mathrm{N}=80)$, uma evidência de validade discriminante: $r(\mathrm{IC} 95 \% \mathrm{BCa})=-0,22(-0,37 ;-0,07)$, $\mathrm{p}=0,046$. A análise em pacientes hipertensos $(\mathrm{N}=61)$ mostrou que o CPM dos anti-hipertensivos correlacionou-se positivamente à adesão e ao controle da pressão arterial, de acordo com os respectivos coeficientes: $\mathrm{r}(\mathrm{IC} 95 \% \mathrm{BCa})$ $=0,70(0,37 ; 0,85), \mathrm{p}<0,001$ e $\mathrm{r}_{\mathrm{b}}(\mathrm{IC} 95 \% \mathrm{BCa})=$ $0,46(0,11 ; 0,72), \mathrm{p}=0,029$.

A despeito da diferença de estrutura interna revelada pela ACP, considerou-se que a equivalência funcional foi satisfatória devido às evidências de equivalência conceitual, de item, semântica e operacional, e de consistência interna e validade.

\section{Discussão}

O questionário CPM-ES-ES foi adaptado transculturalmente ao contexto cultural brasileiro. A versão adaptada foi considerada adequada para medir, no Brasil, o conhecimento sobre medicamentos, uma vez que apresentou equivalência conceitual, de item, semântica, operacional e funcional em relação à versão original, além de evidências satisfatórias de confiabilidade e validade. Houve diferenças nas perguntas incluídas em dois dos quatro componentes identificados pela ACP na versão adaptada em relação à original, evidenciando desconformidades de estrutura interna. 
Tabela 2. Distribuição das variáveis do respondente do número de medicamentos prescritos entre pacientes $(\mathrm{N}=$ 41) e cuidadores $(\mathrm{N}=39)$, São Paulo-SP, 2013-2014.

\begin{tabular}{|c|c|c|}
\hline Variável & Pacientes & Cuidadores \\
\hline Número de indivíduos & 41 & 39 \\
\hline Idade (anos), mediana (IIQ) & $84,0(83,0-87,0)$ & $56,0(49,0-60,0)$ \\
\hline \multicolumn{3}{|l|}{ Sexo, $\mathrm{n}(\%)$} \\
\hline Masculino & $10(24,4)$ & $3(7,7)$ \\
\hline Feminino & $31(75,6)$ & $36(92,3)$ \\
\hline \multicolumn{3}{|l|}{ Nível de instrução, n(\%) } \\
\hline Sem ensino a fundamental incompleto & $25(61,0)$ & $5(12,8)$ \\
\hline Fundamental completo a médio incompleto & $2(4,9)$ & $5(12,8)$ \\
\hline Médio completo a superior incompleto & $5(12,2)$ & $18(46,2)$ \\
\hline Superior completo & $9(21,9)$ & $11(28,2)$ \\
\hline \multicolumn{3}{|l|}{ Rendimento mensal ${ }^{\mathrm{a}}\left(\mathrm{SM}^{\mathrm{b}}\right), \mathrm{n}(\%)$} \\
\hline$\leq 1,0$ & $12(30,0)$ & $7(18,9)$ \\
\hline $1,1-4,0$ & $18(45,0)$ & $17(46,0)$ \\
\hline$>4,0$ & $10(25,0)$ & $13(35,1)$ \\
\hline Medicamentos prescritos, mediana (IIQ) & $5,0(4,0-8,0)$ & $6,0(5,0-8,0)$ \\
\hline \multicolumn{3}{|l|}{ Medicamentos prescritos, $\mathrm{n}(\%)$} \\
\hline $1-4$ & $11(26,8)$ & $9(23,1)$ \\
\hline $5-8$ & $20(48,8)$ & $24(61,5)$ \\
\hline$\geq 9$ & $10(24,4)$ & $6(15,4)$ \\
\hline
\end{tabular}

Tabela 3. Análise de componentes principais inicial e após exclusão da pergunta 8, pacientes e cuidadores, $\mathrm{N}=$ 80, São Paulo-SP, 2013-2014.

\begin{tabular}{lcccccccc}
\hline & \multicolumn{4}{c}{ Pesos fatoriais $^{\mathbf{a}}$} & \multicolumn{5}{c}{ Pesos fatoriais $^{\mathbf{b}}$} \\
\hline Pergunta & 1 & 2 & 3 & 4 & 1 & 2 & 3 & 4 \\
2 (dose) & 0,87 & 0,07 & 0,09 & $-0,04$ & 0,86 & 0,09 & 0,12 & 0,02 \\
3 (frequência) & 0,83 & 0,09 & 0,07 & $-0,09$ & 0,85 & 0,09 & 0,03 & 0,10 \\
4 (duração do tratamento) & 0,84 & 0,14 & 0,13 & $-0,09$ & 0,84 & 0,17 & 0,16 & 0,05 \\
5 (forma de administração) & 0,77 & 0,03 & 0,01 & 0,00 & 0,77 & 0,02 & 0,01 & 0,01 \\
9 (efetividade) & $-0,01$ & 0,86 & 0,13 & $-0,06$ & $-0,03$ & 0,83 & 0,07 & 0,20 \\
1 (indicação) & 0,39 & 0,75 & $-0,14$ & 0,11 & 0,34 & 0,78 & 0,03 & $-0,21$ \\
10 (interações) & $-0,13$ & 0,11 & $-0,72$ & $-0,18$ & $-0,05$ & 0,07 & $-0,89$ & 0,01 \\
11 (conservação) & 0,24 & 0,19 & 0,60 & $-0,28$ & 0,21 & 0,25 & 0,61 & 0,30 \\
7 (reações adversas) & $-0,05$ & 0,05 & 0,71 & $-0,06$ & 0,02 & $-0,14$ & 0,23 & 0,80 \\
6 (precauções) & $-0,06$ & $-0,42$ & $-0,17$ & 0,68 & $-0,12$ & $-0,43$ & 0,10 & $-0,62$ \\
8 (contraindicações) & $-0,09$ & 0,24 & 0,07 & 0,85 & & & & \\
\hline Autovalor & 3,38 & 1,47 & 1,35 & 1,21 & 3,37 & 1,47 & 1,33 & 0,87 \\
Variância explicada (\%) & 30,75 & 13,36 & 12,30 & 11,03 & 33,71 & 14,66 & 13,28 & 8,70 \\
\hline
\end{tabular}

${ }^{\mathrm{a} A n a ́ l i s e ~ i n i c i a l ~ r e a l i z a d a ~ c o m ~ t o d a s ~ a s ~} 11$ perguntas. ${ }^{\mathrm{b}}$ Nova análise realizada com 10 perguntas, após exclusão da pergunta 8.

As sugestões de melhoria semântica foram criadas na intenção de facilitar a avaliação de medicamentos que ainda não foram utilizados, para os quais se sugere realizar a pergunta no futuro. Também facilitam a investigação de medicamentos que não são ingeridos, para os quais se sugere substituir tomar por utilizar. Os farmacêuticos consideraram que estas sugestões não prejudicaram as equivalências obtidas no processo de ATC.

$\mathrm{O}$ uso da tradução literal na maioria das questões qualificou o processo de tradução uma vez que se pressupõe haver correspondência literal entre duas palavras de culturas distintas caso tenham o mesmo significado denotativo ${ }^{26}$. 
Vale destacar que na questão 6 elegeu-se a palavra cuidado por ser considerada de mais fácil entendimento que precaução. Na pergunta 3, a opção por quantas vezes ao dia justificou-se por ser considerada de mais fácil compreensão e mais apropriada ao contexto do uso crônico de medicamentos; esta expressão permitiu obter a informação de frequência mesmo para medicamentos não utilizados diariamente. Na questão 7 , o uso do termo reações adversas foi preferido pois se refere a um evento causado necessariamente pelo medicamento, diferentemente de evento adverso, o qual não possui necessariamente uma relação causal com a farmacoterapia ${ }^{36}$. Além disso, o termo reações adversas já havia sido empregado em questionários brasileiros ${ }^{13,15}$.

A baixa performance da pergunta 8 na ACP pode ter sido consequência da baixa dispersão dos seus escores. A decisão dos autores em excluí-la foi pautada pelo seu índice KMO abaixo de 0,5.A mesma decisão foi tomada na análise conduzida com a versão adaptada ao português europeu, em que foram eliminadas as perguntas 6 e $10^{37}$. As ACP do questionário original ${ }^{16} \mathrm{e} \mathrm{da}$ versão adaptada ao português europeu ${ }^{37}$ utilizaram o critério de Kaiser, pelo qual são extraídos somente componentes com autovalores acima de 1,00 . Na original, foram extraídos quatro componentes, formados respectivamente pelas perguntas: 2 a $5 ; 1$ e 9; 6, 7, 8 e 10; e $11^{16}$. Na análise lusitana, foram extraídos três componentes, contendo respectivamente as perguntas: 2,3 e $5 ; 1,4,9$ e 11 ; e 7 e $8^{37}$. O critério de Kaiser é um dos métodos menos exatos para a escolha do número de componentes pois tende a subestimá- $\mathrm{lo}^{38}$ e somente ser exato quando há menos que 30 perguntas e as comunalidades após a extração são todas maiores que $0,7^{32}$. Portanto, ele não foi usado.

O terceiro e o quarto componentes configuraram-se diferentemente do estudo original, o que pode refletir uma diferença de conduta profissional entre farmacêuticos brasileiros e espanhóis. No Brasil, o conceito de dispensação ainda está vinculado apenas à entrega do medicamento; na Espanha, a dispensação é considerada um suporte para a prática da atenção farmacêutica na medida em que deve prevenir o surgimento de problemas relacionados aos medicamentos ${ }^{39}$. Reações adversas e precauções são a quarta e a sexta informação mais lidas em bulas brasileiras respectivamente. Esse comportamento decorre possivelmente da falta de orientação dessas informações por médicos e farmacêuticos e de acesso a serviços de saúde $^{40}$, o que explicaria o agrupamento dessas perguntas na $\mathrm{ACP}$ com exclusão do item 8.
$\mathrm{O} \alpha$ da versão aqui adaptada foi considerado adequado e superior ao da lusitana $(0,519)^{37}$ e ao da original $(0,677)^{16}$. Os outros estudos de validade do CPM-ES-ES ${ }^{16,37}$ não incluíram testes de validade discriminante ou convergente, de modo que não foi possível efetuar comparações. A correlação negativa entre o conhecimento e o ICFT pode ser explicada pela associação, também negativa, entre a complexidade da farmacoterapia e a adesão medicamentosa, a qual foi observada em pacientes diabéticos ${ }^{41}$ e idosos ${ }^{42}$. Ainda, a correlação positiva aqui encontrada entre o conhecimento de anti-hipertensivos e a sua adesão,atesta o CPM como um conjunto de informações que garante o uso correto dos medicamentos e, portanto, endossa a definição de conhecimento proposta originalmente ${ }^{16}$.

Algumas limitações devem ser consideradas. Os farmacêuticos não utilizaram método quantitativo para investigar a extensão da concordância do sentido denotativo e conotativo entre as versões do questionário criadas durante o processo de obtenção da equivalência semântica, como realizado em outros estudos ${ }^{18}$. Com relação à equivalência de mensuração, a amostra pequena pode ter sido a causa das diferenças encontradas, como já mencionado no estudo de validação lusitano $^{37}$. Além disso,devido às diferenças entre pacientes e cuidadores evidenciadas na Tabela 2 , os resultados poderiam variar caso as análises fossem realizadas separadamente para esses dois grupos. Contudo, acreditamos que a inclusão de pacientes e cuidadores na amostra a aproximou da realidade. Muitas vezes, o paciente não é o responsável pela administração dos medicamentos que utiliza; assim, é importante que o questionário tenha sido adaptado, testado e validado com o objetivo de aceitar respostas de cuidadores.

No questionário original e em sua adaptação para o português europeu, a equivalência de mensuração foi obtida para uma amostra consecutiva de usuários de farmácias comunitárias sem restrições de idade. As idades variaram de 18 a 83 anos na Espanha (média de 46,9) ${ }^{16}$ e de 20 a 87 anos em Portugal, que não declarou a média dos entrevistado ${ }^{37}$. Devido sobretudo à homogeneidade etária da amostra, nosso estudo ficou exposto a vieses de seleção, ausentes nos outros dois estudos ${ }^{16,37}$. Ainda, a falta de validade externa como consequência da ausência de amostra probabilística é comum aos três trabalhos. Por outro lado, a realização das entrevistas em ambulatório aproximou os resultados dos outros dois estudos a medida em que possibilitou investigar tanto medicamentos em uso como aqueles nunca 
usados, comprovar a prescrição do medicamento por meio de prontuário ou receituário - exigido no ato da dispensação - e diferenciar usuários de cuidadores. $\mathrm{O}$ espanhol identificou, por exemplo, $7,5 \%$ de cuidadores ${ }^{16}$ e o lusitano $29,0 \%{ }^{37}$.

O CPM-ES-ES pode ser usado em qualquer paciente, independentemente de suas doenças e medicamentos usados $^{16}$. Embora a amostra tenha apresentado alto grau de especificidade, representado, por exemplo, pela seleção apenas de pacientes com 80 anos ou mais, acreditamos que a característica abrangente do questionário supracitado não foi perdida.

Outra limitação é a de que a análise de componentes realizada foi exploratória e não confirmatória, de modo que não foi possível confirmar a hipótese de que o CPM possui, de fato, quatro domínios. Optou-se pela análise exploratória a fim de estudar como a estrutura interna do questionário seria afetada por nosso processo de ATC, visto que a ATC lusitana produziu uma versão com uma estrutura interna muito diferente da original $^{37}$. Dessa forma, estudos confirmatórios e que incluam um maior número de indivíduos são desejados. Além disso, vale mencionar também que não se estimou a equivalência (índice de concordância interobservadores) ou a estabilidade (confiabilidade teste-reteste) do questionário.

\section{Conclusão}

A versão adaptada transculturalmente do questionário CPM-ES-ES ao português brasileiro para idosos com 80 anos ou mais e seus cuidadores foi obtida nesse estudo, e apresentou equivalência conceitual, de item, semântica, operacional e funcional em relação à original, além de evidências satisfatórias de confiabilidade e validade. Dessa forma, pode ser empregada como instrumento de medida do conhecimento sobre medicamentos que são, ou serão, utilizados em indivíduos independentemente da sua condição clínica, idade ou do tipo e número de medicamentos, no contexto brasileiro.

\section{Colaboradores}

TVN Didone trabalhou na concepção da pesquisa, análise e interpretação dos dados, e redação do manuscrito; P García-Delgado, DO Melo, NS Romano-Lieber e FM Martínez trabalharam na revisão crítica do manuscrito e na aprovação da versão a ser publicada; E Ribeiro trabalhou na concepção da pesquisa, análise e interpretação dos dados, revisão crítica do manuscrito e na aprovação da versão a ser publicada.

\section{Referências}

1. Brasil. Ministério da Saúde (MS). Departamento de Atenção Básica. Portaria GM no 3.916, de 30 de outubro de 1998. Aprova a Política Nacional de Medicamentos. Diário Oficial da União 1998; 10 nov.

2. De Vries TPGM, Henning RH, Hogerzeil HV, Fresle DA. Guide to good prescribing. Geneva: World Health Organization/Action programme on essential drugs; 1994.

3. Bergsten-Mendes G. Uso racional de medicamentos: o papel fundamental do farmacêutico. Cien Saude Colet 2008, 13(Supl.):569-577.

4. Carvalho MFC, Romano-Lieber NS, Bergsten-Mendes G, Secoli SR, Ribeiro E, Lebrão ML, Yeda AOD. Polifarmácia entre idosos do Município de São Paulo Estudo SABE. Rev bras epidemiol 2012; 15(4):817-827.

5. Oliveira MA, Francisco PMSB, Costa KS, Barros MBA. Automedicação em idosos residentes em Campinas, São Paulo, Brasil: prevalência e fatores associados. Cad Saude Publica 2012; 28(2):335-345.

6. Barat I, Andreasen F, Damsgaard EMS. Drug therapy in the elderly: what doctors believe and patients actually do. Br J Clin Pharmacol 2001; 51(6):615-622.
7. Bosch-Lenders D, Maessen DW, Stoffers HE, Knottnerus JA, Winkens B, van den Akker M. Factors associated with appropriate knowledge of the indications for prescribed drugs among community-dwelling older patients with polypharmacy. Age Ageing 2016; 45(3):402-408.

8. Hartholt KA, Val JJ, Looman CW, Petrovic M, Schakel A, van der Cammen TJ. Better drug knowledge with fewer drugs, both in the young and the old. Acta Clin Belg 2011; 66(5):367-370.

9. Tang EOYL, Lai CSM, Lee KKC, Wong RSM, Cheng G, Chan TYK. Relationship between patients' warfarin knowledge and anticoagulation control. Ann Pharmacother 2003; 37(1):34-39.

10. Burge S, White D, Bajorek E, Bazaldua O, Trevino J, Albright T, Wright F, Cigarroa L. Correlates of medication knowledge and adherence: findings from the residency research network of South Texas. Fam Med 2005; 37(10):712-718.

11. Silva T, Schenkel EP, Mengue SS. Nível de informação a respeito de medicamentos prescritos a pacientes ambulatoriais de hospital universitário. Cad Saude Publica 2000; 16(2):449-455. 
12. Fröhlich SE, Dal Pizzol TS, Mengue SS. Instrumento para avaliação do nível de conhecimento da prescrição na atenção primária. Rev Saude Publica 2010; 44(6):1046-1054.

13. Ceccato MGB, Acurcio FA, Bonolo PF, Rocha GM, Guimarães MDC. Compreensão de informações relativas ao tratamento anti-retroviral entre indivíduos infectados pelo HIV. Cad Saude Publica 2004; 20(5):1388-1397.

14. Oenning D, Oliveira BV, Blatt CR. Conhecimento dos pacientes sobre os medicamentos prescritos após consulta médica e dispensação. Cien Saude Colet 2011; 16(7):3277-3283.

15. Dresch AP, Amador TA, Heineck I. Conhecimento dos pacientes sobre medicamentos prescritos por odontólogos no sul do Brasil. Cien Saude Colet 2016; 21(2):475-484.

16. Delgado PG, Garralda MAG, Parejo MIB, Lozano FF, Martínez FM. Validación de un cuestionario para medir el conocimiento de los pacientes sobre sus medicamentos. Aten Primaria 2009; 41(12):661-669.

17. Romero-Sanchez J, Garcia-Cardenas V, Abaurre R, Martínez-Martínez F, Garcia-Delgado P. Prevalence and predictors of inadequate patient medication knowledge. J Eval Clin Pract 2016; 22(5):808-815.

18. Rubio JS, Iglésias-Ferreira P, Delgado PG, Mateus-Santos H, Martínez-Martínez F. Adaptação intercultural para português europeu do questionário "Conocimiento del Paciente sobre sus Medicamentos" (CPM -ES-ES). Cien Saude Colet 2013; 18(12):3633-3644.

19. Herdman M, Fox-Rushby J, Badia X. A model of equivalence in the cultural adaptation of HRQoL instruments: the universalist approach. Qual Life Res 1998; 7(4):323-335.

20. American Psychiatry Association (APA). Diagnostic and Statistical Manual of Mental disorders - DSM-5. $5^{\text {th }}$ ed. Washington: American Psychiatric Association; 2013.

21. Sociedade Brasileira de Cardiologia (SBC), Sociedade Brasileira de Hipertensão (SBH), Sociedade Brasileira de Nefrologia (SBN). VI Diretrizes brasileiras de hipertensão. Arq Bras Cardiol 2010; 95(1):1-51.

22. Aronow WS, Fleg JL, Pepine CJ, Artinian NT, Bakris G, Brown AS, Ferdinand KC, Forciea MA, Frishman WH, Jaigobin C, Kostis JB, Mancia G, Oparil S, Ortiz E, Reisin E, Rich MW, Schocken DD, Weber MA, Wesley DJ. ACCF/AHA 2011 Expert consensus document on hypertension in the elderly. J Am Coll Cardiol 2011; 57(20):2037-2114.

23. Melchiors AC, Correr CJ, Fernández-Llimos F. Tradução e validação para o português do Medication Regimen Complexity Index. Arq Bras Cardiol 2007; 89(4):210-218.

24. Micromedex. [acessado 2016 Fev 28]. Disponível em: http://www-micromedexsolutions-com.ez67.periodicos.capes.gov.br/micromedex2/librarian

25. UpToDate [base de dados]. [acessado 2016 Fev 28]. Disponível em: http://www.uptodate.com/contents/search

26. Reichenheim ME, Moraes CL. Operacionalização de adaptação transcultural de instrumentos de aferição usados em epidemiologia. Rev Saude Publica 2007; 41(4):665-673

27. Beaton DE, Bombardier C, Guillemin F, Ferraz MB. Guidelines for the process of cross-cultural adaptation of self-report measures. Spine (Phila $\mathrm{Pa} 1976$ ) 2000; 25(24):3186-3191.
28. Kaiser HF. An index of factorial simplicity. Psychometrika 1974; 39(1):31-36.

29. Field A. Discovering statistics using IBM SPSS statistics. $4^{\text {th }}$ ed. Thousand Oaks: Sage Publications; 2013.

30. Stevens JP. Applied Multivariate Statistics for the Social Sciences. $4^{\text {th }}$ ed. Hillsdale: Erlbaum; 2002.

31. Furr RM, Bacharach VR. Psychometrics: an introduction. $2^{\text {nd }}$ ed. Thousand Oaks: Sage Publications; 2014.

32. Nunnally JC, Bernstein IH. Psychometric theory. $3^{\text {th }}$ ed. New York: McGraw-Hill; 1994.

33. Portal Regional da BVS - Informação e Conhecimento para a Saúde. Conhecimento do Paciente sobre a Medicação. [acessado 2016 set 22]. Disponível em: http:// pesquisa.bvsalud.org/portal/decs-locator/?lang=pt\&tree_id=N02.421.726.407.229.500.500\&term $=$ conhecimento+do+paciente

34. Brasil. Decreto no 7.872, de 26 de dezembro de 2012. Regulamenta a Lei $n^{\circ} 12.382$, de 25 de fevereiro de 2011, que dispõe sobre o valor do salário mínimo e a sua política de valorização de longo prazo. Diário Oficial da União 2012; 26 dez.

35. Brasil. Decreto no 8.166, de 23 de dezembro de 2013. Regulamenta a Lei $\mathrm{n}^{\circ} 12.382$, de 25 de fevereiro de 2011, que dispõe sobre o valor do salário mínimo e a sua política de valorização de longo prazo. Diário Oficial da União 2013; $24 \mathrm{dez}$.

36. World Health Organization (WHO). The Importance of Pharmacovigilance - Safety Monitoring of Medicinal Products. London: WHO; 2002.

37. Rubio JS, Delgado PG, Ferreira PI, Santos HM, Martínez-Martínez F. Validación del cuestionario de medida del conocimiento del paciente sobre su medicamento adaptado al portugués. Cien Saude Colet 2014; 19(4):1141-1150.

38. Costello AB, Osborne JW. Best practices in exploratory factor analysis: four recommendations for getting the most from your analysis. Practical Assessment, Research \& Evaluation 2005; 10(7):1-9.

39. Angonesi D. Dispensação farmacêutica: uma análise de diferentes conceitos e modelos. Cien Saude Colet 2008; 13(Supl.):629-640.

40. Silva M, Almeida AE, Oliveira AM, Correia CC, Benzatti FP, Fernandes JT, Barbosa GR, Pimenta CP, Costa TMM, Doneida VC. Estudo da bula de medicamentos: uma análise da situação. Rev ciênc farm básica apl 2006; 27(3):229-236.

41. De Vries ST, Keers JC, Visser R, de Zeeuw D, Haaijer-Ruskamp FM, Voorham J, Denig P. Medication beliefs, treatment complexity, and non-adherence to different drug classes in patients with type 2 diabetes. J Psychosom Res 2014; 76(2):134-138.

42. Mansur N, Weiss A, Beloosesky Y. Looking beyond polypharmacy: quantification of medication regimen complexity in the elderly. Am J Geriatr Pharmacother 2012; 10(4):223-229.

Artigo apresentado em 15/03/016

Aprovado em 01/02/2018

Versão final apresentada em 03/02/2018 